ORIGINAL ARTICLE

\title{
Change of nitric oxide in experimental colitis and its inhibition by melatonin in vivo and in vitro
}

\author{
Q Mei, J-M Xu, L Xiang, Y-M Hu, X-P Hu, Z-W Xu
}

Postgrad Med J 2005;81:667-672. doi: 10.1136/pgmj.2004.030817

See end of article for authors' affiliations

.....................

Correspondence to:

Professor J-M Xu, Digestive

Department Of The First

Affiliated Hospital In Anhui

Medical University,

230032, 218 Jixi Road

Hefei City, Anhui Province,

China; XJM1017@yahoo.

com.cn

Submitted

18 November 2004

Accepted 9 March 2005
Aim: To investigate the change of nitric oxide $(\mathrm{NO})$ in rat colitis and its inhibition by melatonin in vivo and in vitro.

Methods: In vivo, rat colitis was established intracolonically with trinitrobenzenesulphonic acid (TNBS) and ethanol. The animals were randomised into five groups: control group, model group, melatonin group $(2.5,5.0,10.0 \mathrm{mg} / \mathrm{kg}$ ), and treated intracolonically with saline, saline and melatonin respectively (once a day, from day 7 after colitis was established to day 28). After the end of the experiment, the mucosal damage index (CMDI) and histology score (HS) were evaluated and the level of myeloperoxidase (MPO) and malondiadehyde (MDA) and NO in the colon tissue were measured. In vitro, the co-culture model of the inflamed colon mucosa (from the colitis) with lipopolysaccharide (LPS), and the colonocytes oxidative injury model by hydroxyl radical, were designed respectively to elucidate the inhibition of NO by melatonin.

Results: After treated with TNBS/ethanol, the extent of CMDI and HS, the levels of MPO, MDA, and NO in the model group, were higher than that in the control group; melatonin ameliorated these parameters effectively. The stimulation of LPS increased the level of NO and MPO and MDA in the co-culture model of inflamed colon mucosa, and melatonin significantly reduced the level of MPO, MDA, and NO. In the coloncyte oxidative injury model by hydroxyl radical, the contents of LDH, MDA, and NO were increased; melatonin reversed this oxidative injury considerably.

Conclusion: This study showed that TNBS/ethanol induced colitis was pharmacologically controlled by melatonin in vivo and in vitro.
! ncreased production of reactive metabolites of nitrogen has a significant pathophysiological role in the development of inflammation. Animal models of intestinal inflammation ${ }^{12}$ and human inflammatory bowel disease (IBD) $)^{3-6}$ are characterised by the increased production of nitric oxide (NO) and the increased expression of the inducible isoform of NO synthase (iNOS). ${ }^{7}$ For example, iNOS is expressed in colon epithelial cells response to proinflammatory stimuli. ${ }^{8-10}$ In an inflammatory focus, NO may react with superoxide anion, resulting in oxidative tissue damage through production of peroxynitrite, ${ }^{11}$ which is believed to mediate many of the destructive effects of NO in colon inflammation. ${ }^{12}$ There are many reports showing that longstanding inflammation of the colon can be ameliorated by the inhibition of iNOS in experimental colitis. ${ }^{7}$

Melatonin, the major product of the pineal gland, plays a fundamental part in the neuroimmunoendocrine system. It was reported that melatonin and melatonin binding sites are found highly concentrated within the intestine. ${ }^{13}$ Furthermore, it has been suggested that the intestine is the major site for extrapineal melatonin activity in the gut. Recently, it was shown that melatonin is a free radical scavenger ${ }^{14-17}$ and protects cells against the damage induced by several oxidative agents including paraquat ${ }^{18}$ and carbon tetrachloride. ${ }^{19}$ Melatonin inhibits the production of NO, and is also a scavenger of peroxynitrite, which is a toxic oxidant formed from the reaction of superoxide and NO under conditions of inflammation..$^{20}$ As a strong antioxidant, melatonin has been reported to play a protective part against colonic mucosal injury. ${ }^{22}$ In murine and rat models of colitis, melatonin also blocked several parameters of gut injury in chemically induced models of colitis. ${ }^{24} 25$

To investigate the role of the NO production in the pathophysiology of experimental colitis, especially the inhibition of this change by melatonin, we designed this study to elucidate the molecular pathways that lead to the protection of melatonin against colitis.

\section{METHODS \\ Animals}

Male Sprague-Dawley rats (mean (SD) 280 (20) g, Experimental Animal Centre of Anhui Medical University, China) were kept in a controlled environment $(20 \pm 1)^{\circ} \mathrm{C}$ and light/dark ( $12 \mathrm{~h}: 12 \mathrm{~h}$ ) cycle for one week and provided with standard rodent chow and water.

\section{Reagents and chemicals}

Melatonin, MTT, lipopolysaccharide (LPS), N-1-naphthylenediamine hydrochloride, O-dianisidine, hexadecyltrimethylammonium bromide, 2,4,6-trinitrobenzene sulphonic acid (TNBS), collagenase were purchased from Sigma Co (USA). Thiobarbituric acid (TBA) and 1,1,3,3-tetraethoxypropane (TEP) were purchased from Fluka (Switzerland). Tripsin, dithiothreitol (DTT), and trypan blue were provided by HuaMei Biotech (China). Bovine serum albumin (BSA) was provided by Bang-Ding Biotech (China). Pronase was provided by Serva. RPMI-1640 medium was provided by Gibco (USA). All other reagents and compounds were purchased from Sigma (USA).

Abbreviations: NO, nitric oxide; TNBS, trinitrobenzenesulphonic acid; $\mathrm{IBD}$, inflammatory bowel disease; CMDI, colon mucosal damage index; MPO, myeloperoxidase; MDA, malondiadehyde; iNOS, isoform of NO synthase; LPS, lipopolysaccharide; TBA, thiobarbituris acid; HBSS, Hank's balanced salt solution 


\section{In vivo experiment}

Experiment protocol

In the TNBS/ethanol induced colitis, rats were randomly divided into five groups: control group, model group, and melatonin groups $(2.5,5.0,10.0 \mathrm{mg} / \mathrm{kg})$. Melatonin dissolved in ethanol/saline $(1 / 100, v / v)$ was given daily as an intracolonic solution of $2.5,5.0$, and $10.0 \mathrm{mg} / \mathrm{kg}$ respectively from day 7 after colitis was established to day 28. In the control and model group of rats, ethanol/saline was given instead of melatonin, respectively.

\section{Induction of colitis}

TNBS/ethanol induced colitis in the rat was established according to Elson et al. ${ }^{26}$ After an overnight fast, each rat was lightly anaesthetised with diethyl ether, and a polyethylene cannula (diameter $4 \mathrm{~mm}$ ) was inserted into the lumen of the colon. The cannula was advanced so that the tip was $8 \mathrm{~cm}$ proximal to the anus. Initially, each rat was lavaged with $2 \mathrm{ml}$ of saline for enema followed by manual palpation of the abdomen to remove any fecal matter. Next, TNBS (100 mg/ $\mathrm{kg}$ ) dissolved in $50 \%$ ethanol $(\mathrm{v} / \mathrm{v})$ was instilled into the colon lumen through the rubber catheter (the final volume $0.25 \mathrm{ml}$ ), and the rat was maintained in a head-down position for 20 seconds to limit expulsion of the solution. In the control group rats, $50 \%$ ethanol was instilled as control.

\section{Assessment of the colitis severity}

At the end of experiment, the tissue of colon $10 \mathrm{~cm}$ proximal to the anus was excised, opened longitudinally, washed in saline buffer, and pinned out on a wax block. Each colon was examined and evaluated by two independent observers. Then colon tissue samples were quickly excised for histological examination and other measurements.

Macroscopic colon damage was scored by two independent observers, described as colonic mucosal damage index (CMDI), according to the following morphological criteria ${ }^{27}$ $0=$ no damage; $1=$ mild hyperaemia and oedema, no erosion or ulcer existing in the mucosa surface; $2=$ moderate hyperaemia and oedema, erosion existing in the mucosa surface; 3 = severe hyperaemia and oedema, necrosis and inflammation and ulcer existing in the mucosa surface, area of the major ulceration extending less than $1 \mathrm{~cm} ; 4=$ severe hyperaemia and oedema, necrosis and ulcer on mucosa, area of the major ulceration extending more than $1 \mathrm{~cm}$.

The colon tissue samples taken for histological examination were fixed overnight in $4 \%$ neutral buffered formalin, processed and sectioned ( $4 \mu \mathrm{m}$ thick), and stained with haematoxylin and eosin. Each colon was examined and evaluated by two independent observers. The assessment criteria of the histopathological score (HS) were modified according to Dieleman $e a^{28}$ using a scoring system: 1-the infiltration of inflammatory cells $(0-3$ : none, slight, moderate, severe); 2-epithelial damage (0-3:none, mucosal, mucosal and submucosal, transmural); 3-the deposition of fibrotin protein (0-1: negative, positive); 4-the submucosa oedema (0-2: no, moderate, severe); 5-the epithelium necrosis (0-2: no, moderate, severe); 6-the epithelium ulcer (0-1: negative, positive).

\section{In vitro experiment}

Experimental protocol and the co-culture model of the inflamed colon mucosa strips from colitis rats ${ }^{29}$ Experimental protocol was divided into control group (coculture of the inflamed colon mucosa strips and vehicle for two hours, then without LPS for 24 hours), model group (coculture of the inflamed colon mucosa strips and vehicle for two hours, then with LPS for 24 hours), melatonin pretreatment group (co-culture of the inflamed colon mucosa strips and melatonin for two hours, then with LPS for 24 hours, melatonin concentration $0.01,0.1,1.0 \mathrm{mmol} / \mathrm{l})$. Colon mucosa strips were obtained from rats with TNBS induced colitis, melatonin dissolved in vehicle (ethanol/saline, 1/100, $\mathrm{v} / \mathrm{v}$ ) at a concentration of $0.01,0.1,1.0 \mathrm{mmol} / \mathrm{l}$ were added to the culture medium and vehicle was also added as control before LPS stimulation.

One week after TNBS/ethanol administration, rat colon was obtained as described before. Colon mucosal specimens were scraped from the marked inflamed part, avoiding ulcerative areas by means of sharp scissors and forceps, the tissue was gently washed and weighed (about $100 \mathrm{mg}$ ), and placed in calcium and magnesium free Hank's balanced salt solution (HBSS) supplemented with $100 \mathrm{IU} / \mathrm{ml}$ penicillin and $100 \mu \mathrm{g} / \mathrm{ml}$ streptomycin, $0.01 \mathrm{mg} / \mathrm{ml}$ trypsin, $10 \mathrm{mmol} / \mathrm{l}$ EDTA, $50 \mathrm{mmol} / \mathrm{l}$ HEPES at $4^{\circ} \mathrm{C}$ for 30 minutes. After collection, they were then placed in 24 well tissue culture plates in $2 \mathrm{ml}$ of RPMI 1640 culture medium for two hours before stimulation with or without $100 \mu \mathrm{g} / \mathrm{ml}$ LPS (at a final concentration of $10 \mu \mathrm{g})$. Melatonin $(0.01,0.1,1.0 \mathrm{mmol} / \mathrm{l})$

Table 2 Effects of melatonin on the content of nitric oxide (NO) in rat colitis

\begin{tabular}{lll}
\hline Group & Dose $(\mathrm{mg} / \mathrm{kg})$ & $\mathrm{NO}_{2}{ }^{-} / \mathrm{NO}_{3}{ }^{-}(\mu \mathrm{mol} / \mathrm{g})$ tissue \\
\hline Control & - & $0.28(0.06)^{* *}$ \\
Model & - & $0.53(0.06)$ \\
Melatonin & 2.5 & $0.40(0.04)^{* *}$ \\
& 5.0 & $0.38(0.02)^{* *}$ \\
& 10.0 & $0.34(0.01)^{* *}$ \\
\hline
\end{tabular}

The treatments were the same as described in table 1. The content of nitric oxide (NO) was measured using a modification of the standard Griess assay and absorbance was read at $540 \mathrm{~nm}$ in a spectrophotometer.

Values are mean (SD) of data obtained from eight rats in each group. ${ }^{* *} p<0.01$ compared with the model group.

Table 1 Effects of melatonin on the value of the colon mucosa damage index (CMDI) and histology score (HS) and the myeloperoxidase (MPO) activity and the malondialdehyde (MDA) concentrations in rat colitis

\begin{tabular}{llllll}
\hline Group & Dose $(\mathrm{mg} / \mathrm{kg})$ & CMDI & HS & MPO (U/g) tissue & MDA (nmol/g) tissue \\
\hline Control & - & $0.0(0.0)^{* *}$ & $0.7(1.2)^{* *}$ & $28.7(20.4)^{* *}$ & $26.5(9.2)^{\star *}$ \\
Model & - & $3.1(1.1)$ & $9.7(0.5)$ & $191.3(32.7)$ & $82.0(21.2)$ \\
Melatonin & 2.5 & $2.3(0.5)$ & $4.5(3.2)^{\star *}$ & $175.8(10.6)$ & $72.2(12.0)$ \\
& 5.0 & $1.7(0.8)^{*}$ & $3.3(0.8)^{\star *}$ & $139.3(37.1)^{* *}$ & $63.5(12.4)^{*}$ \\
& 10.0 & $1.7(0.8)^{*}$ & $2.1(0.8)^{* *}$ & $117.2(28.0)^{* *}$ & $39.2(8.2)^{* *}$ \\
\hline
\end{tabular}

Colonic damage was scored by two independent observers as described in the text. The MPO activity and the MDA concentrations were significantly increased in the model group in comparison with the control group. Melatonin $(2.5,5.0,10.0 \mathrm{mg} / \mathrm{kg}$, once daily) was given intracolonically to the colitis rats (day 7 after the intracolinc TNBS/ ethanol for 21 days). Melatonin treated rats showed a significant reduction in CMDI and HS and MPO activity and MDA concentrations. Values are mean (SD) of data obtained from eight rats in each group. ${ }^{* *} \mathrm{p}<0.01,{ }^{*} \mathrm{p}<0.05$, compared with the model group. 
was also added into the culture medium before exposed to LPS. After 24 hours in culture at $37^{\circ} \mathrm{C}$ in a humidified $5 \%$ $\mathrm{CO}_{2}, 95 \%$ air atmosphere, the tissues were homogenised and the homogenates were centrifuged at $3000 \mathrm{rpm}$ for $10 \mathrm{~min}-$ utes, and the supernatant and pellet were saved on ice. The contents of NO, MDA, and MPO in supernatant were detected as described above.

Experimental protocol and the colonocytes oxidative injury model by hydroxyl radical

Experimental $^{30}$ protocol was divided into control group (COculture of the colonocyte with vehicle for two hours, then without $\mathrm{FeSO}_{4}$ and $\mathrm{H}_{2} \mathrm{O}_{2}$ for three hours), model group (coculture of the colonocyte with vehicle for two hours, then with $\mathrm{FeSO}_{4}$ and $\mathrm{H}_{2} \mathrm{O}_{2}$ for three hours), melatonin pretreatment group (co-culture of the colonocyte with melatonin for two hours, then with $\mathrm{FeSO}_{4}$ and $\mathrm{H}_{2} \mathrm{O}_{2}$ for three hours, melatonin concentration $0.01,0.1,1.0 \mathrm{mmol} / \mathrm{l})$. The colonocytes were obtained from normal rats and resuspended in 24 well tissue culture plates at a final concentration of $1 \times 10^{6} / \mathrm{ml}$ in a culture medium with or without injury by Fenton reaction for two hours, at $37^{\circ} \mathrm{C}$ in a humidified $5 \% \mathrm{CO}_{2}, 95 \%$ air atmosphere. Melatonin dissolved in vehicle (ethanol/ saline, $1 / 100, \mathrm{v} / \mathrm{v}$ ) at a concentration of $0.01,0.1,1.0 \mathrm{mmol} / \mathrm{l}$ was also added to the culture medium before injury by Fenton reaction and vehicle was also added as control. Fenton reaction was exaggerated by supplementation with $\mathrm{FeSO}_{4} 20 \mu \mathrm{l}$ and $\mathrm{H}_{2} \mathrm{O}_{2} 20 \mu \mathrm{l}$ (final concentration was $10 \mathrm{mmol} / \mathrm{l}$, total medium volume was $2.0 \mathrm{ml}$ ) for three hours, then centrifuged at $1000 \mathrm{rpm}$ for three minutes at $4^{\circ} \mathrm{C}$, and the supernatant was saved on ice for the dectection of the contents of $\mathrm{NO}$, the lactate dehydrogenase (LDH) and MDA in supernant was detected as described above.

Colonocytes were isolated according to reference with modification. ${ }^{31-33}$ Briefly, the normal rat colon was excised, washed with oxygenated calcium and magnesium free HBSS supplemented with $100 \mathrm{IU} / \mathrm{ml}$ penicillin and $100 \mu \mathrm{g} / \mathrm{ml}$ streptomycin. Colon mucosal specimens were scraped and cut into pieces $\left(1 \mathrm{~mm}^{3}\right)$ by sharp scissors and forceps, and placed immediately in oxygenated calcium and magnesium free HBSS supplemented with $5 \mathrm{mmol} / \mathrm{l}$ DTT at $4^{\circ} \mathrm{C}$, then centrifuged at $3000 \mathrm{rpm}$ for three minutes, and placed in a conical flask with calcium and magnesium free HBSS (containing $1 \mathrm{~g} / \mathrm{l}$ pronase, $0.01 \mathrm{~g} / \mathrm{l}$ trypsin, $5 \mathrm{mmol} / \mathrm{l}$ EDTA, $50 \mathrm{mmol} / \mathrm{l}$ HEPES (pH 7.4)) for 20 minutes at $37^{\circ} \mathrm{C}$ in a waterbath with shaking at 50 cycles/min, the buffer was saturated with $\mathrm{O}_{2} / \mathrm{CO}_{2}$ (95\%:5\%), then stopped by adding ice cold HBSS. After filtering to remove connective tissue and undigested mucosal mass, the buffer centrifuged at $1000 \mathrm{rpm}$ for three minutes at $4^{\circ} \mathrm{C}$. The pellet was collected and digested with $0.1 \mathrm{~g} / \mathrm{l}$ collagenase again as described above, filtered (gauge in 300-400 $\mu \mathrm{m}$ ) and centrifuged and washed, then resuspended in 24 well tissue culture plates at a final concentration of $1 \times 10^{6} / \mathrm{ml}$ in a culture medium RPMI
1640 with $2.5 \mathrm{~g} / \mathrm{l} \mathrm{BSA}, 2 \mathrm{mmol}$ L-glutamine, $100 \mathrm{IU} / \mathrm{ml}$ penicillin, and $100 \mu \mathrm{g} / \mathrm{ml}$ streptomycin) for two hours at $37^{\circ} \mathrm{C}$ in a humidified $5 \% \mathrm{CO}_{2}, 95 \%$ air atmosphere. Viability of cells was measured by trypan blue dye exclusion as well as by assay of $\mathrm{LDH}_{\mathrm{S}} / \mathrm{LDH}_{\mathrm{T}}$ value. ${ }^{34}$ Cell viability estimated was always greater than $97 \%$. $\mathrm{LDH}_{\mathrm{S}}$ was expressed as the LDH content of a $0.1 \mathrm{ml}$ suspension from the culture medium, $\mathrm{LDH}_{\mathrm{T}}$ was expressed as the $\mathrm{LDH}$ content of a $0.1 \mathrm{ml}$ suspension from the colonocytes homogenates.

\section{Myeloperoxidase (MPO) activity}

MPO activity was determined as an indicator of polymorphonuclear leucocyte accumulation. The colon tissue samples taken for the detection of MPO were homogenised ( $50 \mathrm{~g} / \mathrm{l})$ in ice cold $50 \mathrm{mmol} / \mathrm{l}$ potassium phosphate buffer ( $\mathrm{pH}$ 6.0) containing $0.5 \%$ hexadecyltrimethylammonium bromide. The homogenate was frozen and thawed three times, then centrifuged at $4000 \mathrm{~g}$ for 20 minutes at $4^{\circ} \mathrm{C}$. The level of MPO in supernatant was measured using the $O$-dianisidine method according to Daniel et al. ${ }^{35}$ The rate of change in absorbance was measured spectrophotometrically at $460 \mathrm{~nm}$. MPO activity was expressed in units per gram weight of wet tissue.

\section{Nitric oxide (NO)}

The colon tissue samples were homogenised in ice cold phosphate buffer saline (PBS) ( $\mathrm{pH} 7.4$ ). The homogenates were centrifuged at $4000 \mathrm{~g}$ at $4^{\circ} \mathrm{C}$ for 30 minutes, and the supernatant was stored at $-80^{\circ} \mathrm{C}$ until determination for NO. The content of NO was assessed by colorimetry. ${ }^{36}$

\section{Malondialdehyde (MDA) measurement}

The levels of MDA in the colon were determined as an indicator of lipid peroxidation. The rat colons were rinsed with cold PBS, weighed, and homogenised in $1.15 \% \mathrm{KCl}$ solution using a tissue homogeniser. The supernatants were obtained by centrifugation at $3500 \mathrm{~g}$ of colon homogenates. An aliquot $(500 \mu \mathrm{l})$ of the supernatants was added to a reaction mixture containing $200 \mu \mathrm{l}$ of $8.1 \%$ sodium dodecyl sulphate, $1500 \mu \mathrm{l}$ of $15 \% \mathrm{w} / \mathrm{w}$ trichloroacetic acid, $1500 \mu \mathrm{l}$ of $0.67 \%$ thiobarbituric acid, and $300 \mu \mathrm{l}$ of distilled water. The resulting mixture was boiled for one hour at $95^{\circ} \mathrm{C}$ and then cooled on ice for 15 minutes, the samples were centrifuged at $3000 \mathrm{~g}$ for 10 minutes. Absorbance of the supernatant was measured spectrophotometrically at $535 \mathrm{~nm}$. TEP was as working standard. ${ }^{37}$

\section{Data analysis}

Data were expressed as means (SD), the difference between the means of two groups was evaluated with one way analysis of variance and a p value of less than 0.05 was considered significant.

Table 3 Effects of melatonin in vitro on the content of NO, MPO, and MDA in the inflamed colon from rat colitis with or without stimulation of LPS

\begin{tabular}{lllll}
\hline Group & Dose $(\mathrm{mmol} / \mathrm{l})$ & $\mathrm{MPO}(\mathrm{U} / \mathrm{ml})$ & $\mathrm{MDA}(\mu \mathrm{mol} / \mathrm{ll})$ & $\mathrm{NO}_{2}{ }^{-} / \mathrm{NO}_{3}{ }^{-}(\mu \mathrm{mol} / \mathrm{l})$ \\
\hline Control & - & $4.5(0.7)^{* *}$ & $0.6(0.1)^{* *}$ & $48.9(2.7)^{* *}$ \\
Model & - & $28.0(1.0)$ & $5.6(0.7)^{* *}$ & $156.5(47.0)$ \\
Melatonin & 0.01 & $26.3(0.8)^{*}$ & $3.7(0.2)^{* *}$ & $126.9(1.9)$ \\
& 0.1 & $21.1(1.3)^{* *}$ & $2.5(0.1)^{* *}$ & $110.7(6.5)$ \\
& 1.0 & $19.0(0.3)^{* *}$ & $1.5(0.4)^{* *}$ & $64.5(1.2)^{* *}$ \\
\hline
\end{tabular}

Experimental protocol as described in the text. A colonic segment obtained from rats with TNBS induced colitis was isolated and cultured with LPS. Melatonin at a concentration of $0.01,0.1$, and $1.0 \mathrm{mmol} / \mathrm{l}$ caused a significant reduction of the MPO and MDA levels, melatonin $(1.0 \mathrm{mmol} / \mathrm{l})$ significantly inhibited the $\mathrm{NO}$ production. Values are mean (SD) of data obtained from four rats in each group. ${ }^{* *} p<0.01,{ }^{*} p<0.05$, compared with the model group. 
Table 4 Effects of melatonin in vitro on the value of $\mathrm{LDH}_{\mathrm{S}} / \mathrm{LDH}_{\mathrm{T}}$ and the level of MDA and $\mathrm{NO}$ in the coloncyte oxidative injury model caused by hydroxyl radical generated from the Fenton reaction

\begin{tabular}{lllll}
\hline Group & Dose $(\mathrm{mmol} / \mathrm{I})$ & $\mathrm{LDH}_{\mathrm{S}} / \mathrm{LDH}_{\mathrm{T}}$ & $\mathrm{MDA}(\mu \mathrm{mol} / \mathrm{I})$ & $\mathrm{NO}_{2}{ }^{-} / \mathrm{NO}_{3}{ }^{-}(\mu \mathrm{mol} / \mathrm{I})$ \\
\hline Control & - & $26.3(5.6)^{* *}$ & $0.50(0.06)^{* *}$ & $37.0(1.5)^{* *}$ \\
Model & - & $71.1(7.2)$ & $2.43(0.15)$ & $64.8(0.7)$ \\
Melatonin & 0.01 & $58.9(2.4)^{* *}$ & $1.77(0.03)^{* *}$ & $56.2(1.3)^{* *}$ \\
& 0.1 & $43.7(5.4)^{* *}$ & $1.33(0.04)^{* *}$ & $52.3(0.6)^{* *}$ \\
& 1.0 & $41.2(4.4)^{* *}$ & $1.17(0.02)^{* *}$ & $48.2(1.5)^{* *}$ \\
\hline
\end{tabular}

Experimental protocol designed as described in the text. The coloncyte oxidative injury model caused by hydroxyl radical generated from the Fenton reaction was established. Pretreatment with melatonin reversed this change in the coloncyte oxidative injury model by the reduced content of $N O, L D H$, and MDA. Values are mean (SD) of data obtained from four rats in each group. ${ }^{* *} \mathrm{p}<0.01$ compared with the model group.

\section{RESULTS}

Effects of melatonin on the macroscopic damage score and the microscopic histological score in rat colitis

The macroscopic features of rat colitis showed that the lines-like ulcers were surrounded by thickening inflammed oedema mucosa and seemed to down-develop into the serosal layers. The whole colon wall was becoming severely thickened. The microscopic features of colon included the transmural infiltration of acute and chronic inflammatory cells, noticeable oedema in the submucosa layer, and occurrence of necrosis and ulceration. All these profiles were very similar to the clinical changes of human IBD.

The CMDI and HS in the model group (3.1 (1.1) and 9.7 (0.5), respectively) were all higher than that in the control group $\left(\begin{array}{lll}0.0 & (0.0)\end{array}\right)$ and 0.7 (1.2), respectively). Administration with melatonin intracolon at different doses $(2.5,5.0,10.0 \mathrm{mg} / \mathrm{kg})$ inhibited the extent of inflammation and prevented the mucosa injury, minimised the ulceration area, and alleviated the colitis symptoms. It was supported by the reduction of the values of CMDI and HS in colon (table 1).

\section{Effects of melatonin on the MPO activity and the MDA concentrations in rat colitis}

Colitis caused by TNBS/ethanol was also characterised by an increase in MPO activity (191.3 (32.7) U/g tissue in the model group compared with 28.7 (20.4) U/g tissue in the control group, respectively). Colonic mucosal MPO activity of rats treated with melatonin showed a significant decrease in a dose dependent fashion compared with the model group (72.2 (12.0), 63.5 (12.4), 39.2 (8.2) U/g tissue, respectively). Correspondingly with the increase in colon MPO activity, the increase of MDA concentrations showed an increase in the colon lipid peroxidation $(26.5(9.2) \mathrm{nmol} / \mathrm{g}$ tissue in the model group compared with $82.0(21.2) \mathrm{nmol} / \mathrm{g}$ tissue in the control group, respectively). Intracolonic instillation of melatonin in a dose of $2.5,5.0$, and $10.0 \mathrm{mg} / \mathrm{kg}$ daily inhibited the colon mucosal oxidative injury effectively (72.2 (12.0), 63.5 (12.4), 39.2 (8.2) nmol/g tissue, respectively) (table 1 ).

Effects of melatonin on the content of NO in rat colitis A striking increase in the content of $\mathrm{NO}$ was seen in the inflamed colon $(0.28(0.06) \mu \mathrm{mol} / \mathrm{g}$ tissue in the model group compared with $0.53(0.06) \mu \mathrm{mol} / \mathrm{g}$ tissue in the control group, respectively). This overproduction of NO has been proposed strongly as a pathogenic factor in colitis. Administration with melatonin $(2.5,5.0$, and $10.0 \mathrm{mg} / \mathrm{kg})$ intracolonically suppressed the increase of NO completely $(0.40(0.04), 0.38$ (0.02), 0.34 (0.01) $\mu \mathrm{mol} / \mathrm{g}$ tissue, respectively), and inhibited the extent of the colonic inflammation and prevented the colon mucosa injury (table 2).
Effects of melatonin in vitro on the content of NO in the inflamed colonic mucosa from rat colitis stimulated by LPS

A colonic segment obtained from rats with TNBS induced colitis was isolated and cultured with LPS for determination of the NO, MPO, and MDA production. The stimulation of LPS elicited an inflammatory response that was characterised by increased level of NO, MPO, and MDA (48.9 (2.7) $\mu \mathrm{mol} / \mathrm{l}$, $4.5(0.7) \mathrm{U} / \mathrm{ml}, 0.6(0.1) \mu \mathrm{mol} / \mathrm{l}$ respectively in the control group, compared with 156.5 (47.0) $\mu \mathrm{mol} / \mathrm{l}, 28.0$ (1.0) U/ml, $5.6(0.7) \mu \mathrm{mol} / \mathrm{l}$ respectively in the model group). Melatonin treatment at dose of $0.01,0.1,1.0 \mathrm{mmol} / \mathrm{l}$ also caused a significant reduction of the MPO levels $(26.3(0.8), 21.1$ (1.3), $19.0(0.3) \mathrm{U} / \mathrm{ml}$, respectively) and the MDA levels (3.7 (0.2), $2.5(0.1), \quad 1.5(0.4) \mu \mathrm{mol} / \mathrm{l}$, respectively), melatonin ( $1.0 \mathrm{mmol} / \mathrm{l}$ ) significant inhibited the NO levels, this result was accordance with the results showed in vivo study (table 3).

\section{Effects of melatonin on the content of NO in the coloncyte oxidative injury model caused by hydroxyl radical generated from the Fenton reaction}

A large amount of hydroxyl radical, which was produced by supplemented $\mathrm{FeSO}_{4}$ and $\mathrm{H}_{2} \mathrm{O}_{2}$, directly injured the coloncyte from normal rats. It was noted that the value of $\mathrm{LDH}_{\mathrm{S}} / \mathrm{LDH}_{\mathrm{T}}$ and the MDA level, was $71.1(7.2) \%$ and $2.43(0.15) \mu \mathrm{mol} / \mathrm{l}$ in the model group respectively, and was more higher than that in the control group (26.3 (5.6)\% and $0.50(0.06) \mu \mathrm{mol} / \mathrm{l}$, respectively). This showed that the formation of NO by coloncyte was associated with the diminished cell viability, and was shown with the evidence of the increased content of LDH and MDA in this model. Pretreatment with melatonin reversed this change in the coloncyte oxidative injury model (table 4).

\section{DISCUSSION}

Although the precise aetiology and the pathogenesis of IBD remains to be elucidated, there is a growing body of both experimental and clinical evidence to suggest that longstanding gut inflammation is associated with increased production of reactive metabolites of nitrogen and increased expression of the iNOS. ${ }^{438}{ }^{39}$ Increased NO synthesis by the iNOS in the inflamed colon may play a key part in the aggravation of the inflammation and the tissue injury. Melatonin has been regarded as a strong antioxidant, also as a peroxynitrite scavenger, and its anti-inflammatory properties have been shown in a variety of experimental models. ${ }^{22}{ }^{23}$ Our aim in this study was to investigate the antiinflammatory effects of melatonin in experimental colitis induced by TNBS/ethanol.

In this study, chronic immunological colitis was induced by intracolonic administration of TNBS in 50\% ethanol-this was a well established model of experimental colitis. Our results showed that melatonin dose dependently decreased 
macroscopic and microscopic colonic damage, reduced the severity of colitis such as the neutrophil infiltration, and the oedema formation. Also melatonin noticeably suppressed the MPO activity in colon mucosa and inhibited the neutrophil mediated mucosal injury in the gut.

Meanwhile, these results showed that the development of colon inflammation was closely associated with increased NO production and neutrophil granulocyte infiltration. Melatonin restored the nitrate and the lipid peroxide malondialdehyde towards normal levels. Our findings were consistent with several studies showing attenuated colonic injury by melatonin in different models of $\mathrm{IBD}^{22}{ }^{23}$ The observed anti-inflammatory effect of the melatonin may be attributed partly to its antioxidant property such as eliminating $\mathrm{NO}$ effectively in the inflamed colon.

On the other hand, the source of the raised NO generation in the colonic mucosa has not been exactly identified. There is some evidence that macrophages, endothelial cells, and epithelial cells may also contribute to the NO generation in inflamed tissues. ${ }^{38-40}$ In accordance with these findings, we designed and constructed the co-culture model of the inflamed colon mucosa tissues to investigate the effect of melatonin on the NO production. This model was composed with the colon epithelial cells and the endothelial cells and the macrophages, representing the important sources of NO and other potentially injurious free radicals. After triggered by LPS, the production of the NO, MPO, and MDA were the manifestations of the colon inflammation, melatonin treatment also caused a significant reduction of the MPO activity and lipid peroxidation and inhibited NO levels in the coculture model of the inflamed colon mucosa stimulated by LPS.

A combination of $\mathrm{NO}$ with superoxide anion may yield the highly toxic species, peroxynitrite. Gilad et al showed that melatonin dose dependently inhibits the peroxynitrite mediated oxidant processes. This action may contribute to the antioxidant and anti-inflammatory effects of melatonin in various pathophysiological conditions. ${ }^{20}$ Pretreatment with melatonin reduced the coloncyte oxidative injury by inhibiting NO production and peroxynitrite formation in vitro. ${ }^{43}$ So we established the coloncyte oxidative injury model caused by hydroxyl radical generated from the Fenton reaction, and showed that NO may react with superoxide anions, leading to the coloncytes oxidative injury. Pretreatment with melatonin reduced this change effectively. These results suggested that $\mathrm{NO}$ and the peroxynitrite were also likely to affect the colon epithelial function. Blocking the formation of NO and the peroxynitrite by melatonin was helpful to afford the coloncyte protection.

So in this study, we evaluated the anti-injury effect of melatonin in two models in vitro including the co-culture model of the inflamed colon mucosa from colitis rats and the coloncyte oxidative injury model by hydroxyl radical. Part of these anti-injury effects of melatonin may be commonly related to an inhibition of NO production, while another part may be related to scavenging oxyradical and peroxynitrite. Therefore, inhibition of NO production serves as a novel experimental approach to treat gut inflammation.

In conclusion, there are increasingly amounts of both experimental and clinical data showing that longstanding colon inflammation is associated with the increased production of NO. We showed that melatonin attenuates the colon injury macroscopically and microscopically, reduces the MPO activity, and ameliorates the degree of lipid peroxidation of the colon in the colitis models. Melatonin was an effective scavenger of the hydroxyl radical and the peroxyl radical, ${ }^{44}$ and stimulated some important antioxidative enzymes such as superoxide dismutase. ${ }^{22}{ }^{23}$ Melatonin also acted as a peroxynitrite scavenger and protected the cultured colon cells in vitro. So the mechanism of the observed antiinflammatory effects of melatonin theoretically may be related to eliminated $\mathrm{NO}$, oxyradicals, or a combination of these. These findings support the view that melatonin exert potent anti-inflammatory effects and that this agent may be useful in the treatment of IBD.

\section{Authors' affiliations}

Q Mei, J-M Xu, L Xiang, Y-M Hu, X-P Hu, Z-W Xu, Digestive Department of First Affiliated Hospital of Anhui Medical University, Heifei, China

Funding: none.

Conflicts of interest: none.

\section{REFERENCES}

1 Miller MJ, Thompson JH, Zhang XJ, et al. Role of inducible nitric oxide synthase expression and peroxynitrite formation in guinea pig ileitis. Gastroenterology 1995;109:1475-83.

2 Rachmilewitz D, Karmeli F, Okon E, et al. Experimental colitis is ameliorated by inhibition of nitric oxide synthase activity. Gut 1995;37:247-55.

3 Kimura H, Miura S, Shigematsu T, et al. Increased nitric oxide production and inducible nitric oxide synthase activity in colonic mucosa of patients with active ulcerative colitis and Crohn's disease. Dig Dis Sci 1997;42:1047-54

4 Lundberg JON, Hellström PM, Lundberg JM, et al. Greatly increased luminal nitric oxide in ulcerative colitis. Lancet 1994;344:1673-4.

5 Rachmilewitz D, Stamler JS, Bachwich D, et al. Enhanced colonic nitric oxide generation and nitric oxide synthase activity in ulcerative colitis and Crohn's disease. Gut 1995;36:718-23.

6 Singer II, Kawka DW, Scott S, et al. Expression of inducible nitric oxide synthase and nitrotyrosine in colonic epithelium in inflammatory bowel disease. Gastroenterology 1996;111:871-85.

7 Miller MJS, Sadowska-Krowicka H, Chotinaruemol S, et al. Amelioration of chronic ileitis by nitric oxide synthase inhibition. J Pharmacol Exp Ther 1992;264:11-16.

8 Kolios G, Rooney N, Murphy CT, et al. Expression of inducible nitric oxide synthase activity in human colon epithelial cells: modulation by $\mathrm{T}$ lymphocyte derived cytokines. Gut 1998;43:56-63.

9 Salzman AL, Eaves-Pyles T, Linn SC, et al. Bacterial induction of inducible nitric oxide synthase in cultured human intestinal epithelial cells. Gastroenterology 1998;114:93-102.

10 Lähde M, Korhonen R, Moilanen E. Regulation of nitric oxide production in cultured human T84 intestinal epithelial cells by nuclear factor-kappa Bdependent induction of inducible nitric oxide synthase after exposure to bacterial endotoxin. Aliment Pharmacol Ther 2000;14:945-54.

11 Grisham MB, Jourd'Heuil D, Wink DA. Nitric oxide. I. Physiological chemistry of nitric oxide and its metabolites: implications in inflammation, Am J Physiol 1999;39:G315-21.

12 McCafferty DM. Peroxynitrite and inflammatory bowel disease. Gut 2000;46:436-9.

13 Bubenik GA. Localization, physiological significance and possible clinical implication of gastrointestinal melatonin. Biol Signals Recept 2001;10:350-66.

14 Reiter RJ, Melchiorri D, Sewerynek E, et al. A review of the evidence supporting melatonin's role as an antioxidant. J Pineal Res 1995;18:1-11.

15 Marshall KA, Reiter RJ, Poeggeler B, et al. Evaluation of the antioxidant activity of melatonin in vitro. Free Rad Biol Med 1996;21:307-15.

16 Stasica P, Ulanski P, Rosiak JM. Melatonin as a hydroxyl radical scavenger. $J$ Pineal Res 1998;25:65-6.

17 Sewerynek E, Abe M, Reiter RJ, et al. Melatonin administration prevents lipopolysaccharide-induced oxidative damage in phenobarbital-treated animals. J Cell Biochem 1995;58:436-44.

18 Melchiorri G, Reiter RJ, Attia AM, et al. Potent protective effect of melatonin on in vivo paraquat-induced oxidative damage in rats. Life Sci 1994;56:83-9.

19 Daniels WMU, Reiter RJ, Melchiorri D, et al. Melatonin counteracts lipid peroxidation induced by carbon tetrachloride but does not restore glucose-6phosphatase activity. J Pineal Res 1996;19:1-6.

20 Gilad E, Cuzzocrea S, Zingarelli B, et al. Melatonin is a scavenger of peroxynitrite. Life Sci 1997;60:PL169-74.

21 Cuzzocrea S, Costantino G, Caputi AP. Protective effect of melatonin on cellular energy depletion mediated by peroxynitrite and poly (ADP-Ribose) synthetase activation in a non septic shock model induced by zymosan in the rat. J Pineal Res 1998;25:78-85.

22 Mei Q, Yu JP, Xu JM, et al. Melatonin reduces colon immunological injury in rats by regulating activity of macrophages. Acta Pharmacol Sin 2002;23:882-6.

23 Dong WG, Mei Q, Yu JP, et al. Effects of melatonin on the expression of iNOS and COX-2 in rat models of colitis. World J Gastroenterol 2003;9:1307-11.

24 Cuzzocrea S, Mazzon E, Serraino I, et al. Melatonin reduces dinitrobenzene sulfonic acid-induced colitis. J Pineal Res 2001;30:1-12.

25 Pentney PT, Bubenik GA. Melatonin reduces the severity of dextran-induced colitis in mice. J Pineal Res 1995;19:31-9.

26 Elson CO, Sartor RB, Tennyson GS, et al. Experimental models of inflammatory bowel disease. Gastroenterology 1995;109:1344-67. 
27 Morris GP, Beck PL, Herridge MS, et al. Hapten-induced model of chronic inflammation and ulceration in the rat. Gastroenterology 1989;96:795-803.

28 Dieleman LA, Palmen MJ, Akol H, et al. Chronic experimental colitis induced by dextran sulfate sodium (DSS) is characterized by Th1 and Th2 cytokines. Clin Exp Immunol 1998;1 14:385-91.

29 Wardle TD, Hall L, Turnberg LA. Use of coculture of colonic mucosal biopsies to investigate the release of eicosanoids by inflamed and uninflamed mucosa from patients with inflammatory bowel disease. Gut 1992;33:1644-51.

30 Hata Y, Kawabe T, Hiraishi H, et al. Hydrogen peroxide-mediated cytotoxicity to cultured colonic epithelial cells. Life Sci 1997;60:2221-30.

31 Baten A, Sakamoto K, Shamsuddin AM. Long-term culture of normal human colonic epithelial cells in vitro. FASEB J 1992;6:2726-34.

32 Roediger WEW, Truelove SC. Method of preparing isolated colonic epithelial cells (colonocytes) for metabolic studies. Gut 1979;20:484-8.

33 Masola B, Evered DF. Preparation of rat enterocyte mitochondria. Biochem J 1984;218:441-7.

34 Bergmeyer HU, ed. Methods of enzymatic analysis. New York: Academic Press, 1974:3.

35 Daniel R, Philip LS, Lester WS, et al. Inflammatory mediators of experimental colitis in rats. Gastroenterology 1989;97:326-37.
36 Grisham MB Johnson GG Lancaster JR Jr. Quantitation of nitrate and nitrite in extracellular fluids. Methods Enzymol 1996;268:237-46.

37 Ohkawa H, Ohishi N, Yagi K. Assay for lipid peroxides in animal tissues by thiobarbituric acid reaction. Anal Biochem 1979;95:351-358.

38 Middleton SJ, Shorthouse M, Hunter JO. Increased nitric oxide synthesis in ulcerative colitis. Lancet 1993;341:465-6.

39 Rachmilewitz D, Stamler JS, Bachwich D, et al. Enhanced colonic nitric oxide generation and nitric oxide synthase activity in ulcerative colitis and Crohn's disease. Gut 1995:36:718-23.

40 Grisham MB, Specian RD, Zimmerman TE. Effects of nitric oxide synthase inhibition on the pathophysiology observed in a model of chronic granulomatous colitis. J Pharmacol Exp Ther 1994;271:1114-21.

41 Nathan C. Nitric oxide as a secretory product of mammalian cells. FASEB J 1992:6:3051-64.

42 Tepperman BL, Brown JF, Whittle BJR. Nitric oxide synthase induction and intestinal epithelial cell viability in rats. Am J Physiol 1993;265:G214-18.

43 Hata $Y$, Kawabe T, Hiraishi $H$, et al. Antioxidant defenses of cultured colonic epithelial cells against reactive oxygen metabolites. Eur J Pharmacol 1997:321:113-19.

44 Reiter RJ. The role of the neurohormone melatonin as a buffer against macromolecular oxidative damage. Neurochem Int 1995;27:453-60.

\section{CORRECTION}

doi: 10.1136/pgmj.2004.030825corr 1

There were some editorial errors in this article by Dr Guo and others (2005;81:526-9). The data for the second to last row in table 2 should have read for column T/T 7 (36.9 [not 2 (10.5)]; for column C/C 2 (10.5) [not 7 (36.9)]; for column T 24 (63.2) [not 14 (36.8)]; and for column C 14 (36.8) [not 24 (63.2)]. The data for the last row should have read for column T/T 15 (34.1) [not 2 (4.5)]; for column C/C 2 (4.5) [not 15 (34.1)]; for column T 57 (64.8) [not 31 (35.2)]; and for column C 31 (35.2) [not 57 (64.8)].

\section{CORRECTION}

doi: 10.1136/pgmj.2004.024463corrl

An author's error occurred in this review by Dr Bycroft and others (2005;81:232-5). The name of the third author should have read $\mathrm{E}$ A L Chung [not E A L Choong]. 